\title{
Pivotal Role of Spin in Celestial Body Motion Mechanics: Prelude to a Spinning Universe
}

\author{
Puthalath Koroth Raghuprasad \\ Independent Researcher, Odessa, TX, USA \\ Email: pkrp12@gmail.com
}

How to cite this paper: Raghuprasad, P.K. (2021) Pivotal Role of Spin in Celestial Body Motion Mechanics: Prelude to a Spinning Universe. Journal of High Energy Physics, Gravitation and Cosmology, 7, 98-122.

https://doi.org/10.4236/jhepgc.2021.71005

Received: March 23, 2020

Accepted: December 19, 2020

Published: December 22, 2020

Copyright $\odot 2021$ by author(s) and Scientific Research Publishing Inc. This work is licensed under the Creative Commons Attribution International License (CC BY 4.0).

http://creativecommons.org/licenses/by/4.0/

\section{Abstract \\ This is the final article in our series dealing with the interplay of spin and} gravity that leads to the generation, and continuation of celestial body motions in the universe. In our prior studies we focused on such interactions in the elementary particles, and in the celestial bodies in the solar system. Foremost among the findings was that, along with gravity, matter at all levels exhibits axial spin. We further noted that all freestanding bodies outside our solar system, including the largest such units, the stars and galaxies also spin on their axes. Also, the axial rotation speed of planets in our solar system has a linear positive relationship to their masses, thus hinting at its fundamental and autonomous nature. We have reported that this relationship between the size of the body and its axial rotation speed extends to the stars and even the galaxies. Next, all congregations of matter spin on their axes in the counterclockwise direction; all satellites orbit their mother bodies also in the counterclockwise direction, i.e.: in our solar system, with only rare exceptions, the satellite bodies follow the mother bodies' axial rotation. This relationship exists also in the case of the rings of planets, the asteroids and the Kuiper belt bodies, as well as the stars and their galaxies. We also noted the intricate involvements between spin and gravity in the exquisite phenomena of synchronous and negative rotations of planets and some satellites; we have explained in detail how these two phenomena occur. The closest large moons of the gas and ice giants and the earth's moon exhibit synchronous rotation. In this paper we present evidence that these synchronously rotating satellite bodies' axial rotation speed is closely related to the size and the axial rotation speeds of their respective mother bodies. In the satellites that follow a non-synchronous rotation (most of the planets and their satellites) the satellites' own rotation speed usually dominates. In all these rotational/orbital motions, we believe, the axial rotation and gravity collaborate with the resultant centrifugal force, which prevents the satellite bodies from crashing into the mother bodies. We have inferred from the above observations that the 
axial spin is a fundamental property of matter, akin to gravity, electromagnetism, and strong and weak nuclear forces. This inherent property of matter to spin on its axis is what initiates all celestial body motions and makes such motions perpetual. The lateral motions of stars within the galaxies, are also influenced by the sizes of the stars; the larger the star, the faster it moves radially. Similarly, the larger the spiral galaxy, the faster it rotates on its axis. We extrapolate from these observations that the axial rotational speeds of galaxies affect their motion in space as well, that this is circumferential, and we predict this will also be in the counterclockwise direction. This lateral movement of the galaxies will give the appearance of the whole universe spinning on its axis.

\section{Keywords}

Axial Rotation, Centrifugal Force, Counterclockwise, Gravity, Neutron Stars, Proto-Planetary Disk, Spin

\section{Introduction}

In a prior article we discussed in detail the phenomenon of synchronous rotation in the major satellites of the gas and ice giants and the earth's only moon [1]. We explained how the gravitational and rotational influences from the mother bodies both determine the direction and speeds of orbits of their satellites. We also explained how this orbital motion in the counterclockwise direction will bring about the synchronous rotation. Briefly, as the closest satellite bodies orbit the mother bodies, their front ends will encounter a tug from the gravitational pull from the mother. This augments the axial rotation speeds of the satellites, while still maintaining the counterclockwise direction of the axial rotation of the satellites. In the same paper we addressed the other interesting phenomenon, that of negative or reverse rotation of bodies whose axes are tilted more than 120 degrees; these are Venus and the dwarf planet Pluto and many of the most peripheral satellites of the gas giants. Here, we offered the explanation that the same gravitational pull from the mother bodies acting on the satellite's front ends as well and since the satellite bodies are literally inverted, while still maintaining their rotation in the counterclockwise direction, they encounter an influence from the mother body to rotate in the reverse direction. This conflict leads to extreme slowing of the rotation speed of the satellites and the appearance of "negative rotation".

In our next article [2] we presented data on the linear, positive relationship between the sizes of the regularly rotating planets (Earth, Mars, Jupiter, Saturn, Uranus and Neptune) and their axial rotation speeds, equatorial radii and a non-linear but positive relationship with the degree of surface gravity, and the generation of magnetism. We also showed that in those planets that rotate slowly (Mercury, Venus and Pluto), the equatorial and polar radii are equal, and an ab- 
sence of magnetism in the latter two. Further, we noted that all celestial bodies rotate on their axes and carry their satellites in the same direction. Some of these data are so crucial to the central theme of this paper; we have included them in this paper (Figure 1, Table 1) as well.

In our current paper we present data that extend our observations in our prior papers, to the positive influence of the mother bodies' axial rotation speed on the axial rotation speed of the synchronously rotating satellites of the gas and ice giants of the solar system; this effect is in addition to the augmentation of the orbital speed of the close-by satellites. Other data we present deal with the status of the other solar system bodies as well as the axial rotation and radial velocity of stars and spiral galaxies. We note with interest that stars and even galaxies rotate on their axes in the counterclockwise direction and that even at these levels, the larger the body or conglomeration of bodies, the faster they rotate. Thus, we can conclude with confidence that our initial belief that spin of matter is crucial to the onset of celestial body motion mechanics is correct and it is also the reason why such motions remain perpetual.

\section{Materials and Observations}

We searched the astronomical/astrophysical literature in print and online of relevance to celestial body motions that have been published over the past century. Most useful data were found on the website of NASA, even though their focus was not necessarily from the standpoint of axial spin/orbital motions per se, we were able to find many nuggets and many segments of the data we presented were calculated from the data available on NASA's website. To our surprise, there was a wealth of information to support our ideas, and the picture that we are able to paint explains most of the observed behaviors of astronomical bodies and units. It is comforting to note that, just with the data that we have obtained, we realized that to explain celestial body motions, we did not need to resort to any unknown or unknowable forces, such as "dark matter", "negative energy" or "dark energy" and so on. Some of the data we are presenting below have been published but they are essential for our arguments for the main theme of this paper; thus, we have re-presented them below at the appropriate sections.

The data on stars and galaxies that we were particularly interested in, i.e. their axial rotation rates and their movement in space, and relating them to the respective body's mass and size, were difficult to gather. This difficulty may have been due to two reasons. First, the bodies are situated at such vast distances that data were either not available or they were unreliable. Second, the researchers were not paying special attention to rotational activities, as the concept of "conservation of angular momentum" is so ingrained in scientific circles. (We explain this issue in more detail in the appropriate section later). However, even with the meager data available, we could still find sufficient material to support our contention that even at the levels of stars and galaxies also, the cooperative interactions between gravity and spin remain operative. 


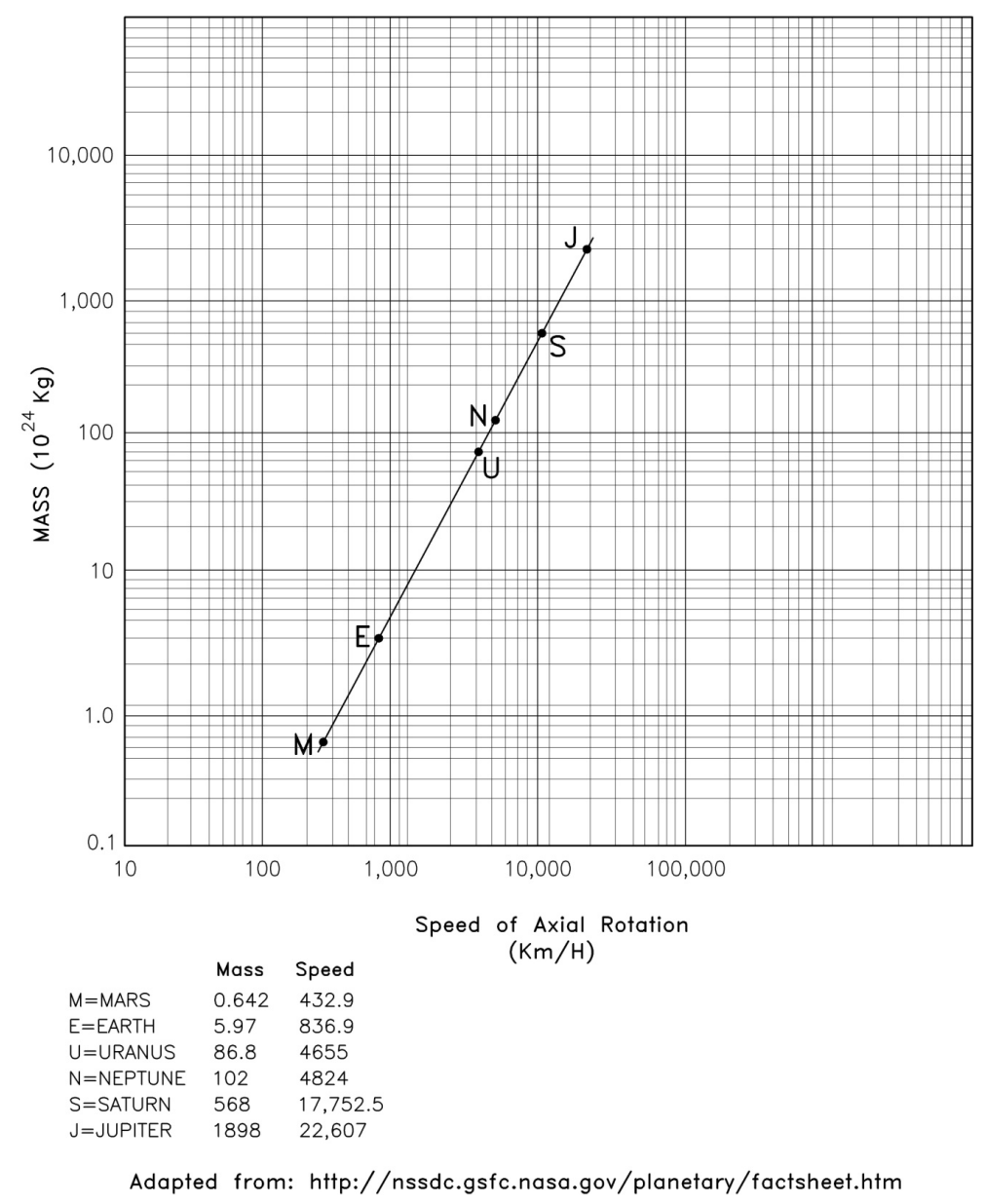

Figure 1. Correlation between mass and speed of axial rotation.

This figure compares the masses of the regularly rotating planets in our solar system, with their axial rotation speeds. For obvious reasons, the two negatively rotating planets (Venus, and the dwarf planet Pluto) and the highly unusual, Mercury were not included in this comparison. It clearly shows a linear relationship: the larger the body, the faster the axial rotation. This shows the fundamental and independent nature of axial rotation of celestial bodies, here represented by these planets. If, on the other hand, this tendency to rotate were derived from the solar nebula at its inception, one would expect all bodies to retain the same rotation rates or, the larger bodies to rotate slower and the smaller ones faster. Only an autonomous, inherent tendency of matter to spin on its axis can explain the above phenomenon where larger bodies rotate faster and smaller ones rotate slower, much like the degree of gravity of celestial bodies, which also increases with the mass of the body. In our paper [1], we have offered our explanation why Uranus, which rotates negatively, is not slowed down; therefore, it was included in this figure, and it does conform to our expectations. This topic is revisited later in Discussion, at the appropriate section. Also, we touch upon the motion characteristics of stars and galaxies, also later in this Materials section and discussed further in Discussion. 
Table 1. Equatorial radii compared to polar radii of planets, and axial rotation rates, as well as gravitation, and the presence of magnetism in the solar system bodies.

\begin{tabular}{|c|c|c|c|c|c|c|c|c|c|}
\hline & Mercury & Venus & Earth & Mars & Jupiter & Saturn & Uranus & Neptune & Pluto \\
\hline $\begin{array}{c}\text { Eq. Rad } \\
(\mathrm{km})\end{array}$ & 2439.7 & 6051.8 & 6378.1 & 3396.2 & 71,492 & 60,268 & 25,559 & 24,764 & 1188 \\
\hline $\begin{array}{l}\text { Polar Rad } \\
\quad(\mathrm{km})\end{array}$ & 2439.7 & 6051.8 & 6356.8 & 3376.2 & 66,854 & 54,364 & 24,973 & 24,341 & 1188 \\
\hline $\begin{array}{l}\text { Sidereal Rotation } \\
\text { (Hrs.) }\end{array}$ & 1407.6 & $-5832.6^{\star}$ & 23.9 & 24.6 & 9.93 & 10.7 & $-17.2^{\star}$ & 16.1 & $-153.3^{\star}$ \\
\hline $\begin{array}{l}\text { Axial Rotation Speed } \\
\qquad(\mathrm{km} / \mathrm{h})\end{array}$ & 10.88 & 6.52 & 1677 & 867 & 45,255 & $35,550.6$ & 9319 & 10,231 & 49 \\
\hline $\begin{array}{l}\text { Gravity } \\
\left(\mathrm{m} / \mathrm{s}^{2}\right)\end{array}$ & 3.7 & 8.9 & 9.8 & 3.7 & 24.8 & 10.44 & 8.7 & 11 & 0.7 \\
\hline Magnetism & Yes & No & Yes & No & Yes & Yes & Yes & Yes & Unknown \\
\hline
\end{tabular}

"Negative rotation" means axial rotation opposite in direction to most of the planets and the sun. Reproduced with kind permission, Physics Essays 26(2013), Vol 26, No 2, pp 331-338. Adapted from https://nssdc.gsfc.nasa.gov/planetary/factsheet/ (Oct. 2019).

Note the increase in the equatorial radius compared to the polar radius, and magnetism in those planets that have rapid axial rotation rates. In contrast, those planets with slow axial rotations (Mercury, Venus and Pluto, printed in bold) display equal equatorial and polar radii (i.e., show no equatorial bulges). There is also a hint that magnetism in planets requires fast axial rotation (see Venus); however, Mercury is magnetic probably due to its high iron content and proximity to Sun. The reason for Mars not having magnetism is not clear but recent findings of rocks on Mars retaining some magnetism indicates that the planet had global magnetism in the distant past [3] [4].

Table 2(a). Planetary axial rotation rates vs. satellites' orbital speeds (Synchronously rotating satellites).

\begin{tabular}{|c|c|c|c|c|c|c|}
\hline \multicolumn{3}{|c|}{ Planets } & \multicolumn{4}{|c|}{ Satellites } \\
\hline & $\begin{array}{c}\text { Mass } \\
\left(10^{24} \mathrm{~kg}\right)\end{array}$ & $\begin{array}{c}\text { Axial } \\
\text { Rot. } \\
\text { Speed } \\
(\mathrm{km} / \mathrm{h})\end{array}$ & & $\begin{array}{c}\text { Dist. } \\
\text { from } \\
\text { Mother } \\
\left(10^{3} \mathrm{~km}\right)\end{array}$ & Mass* & $\begin{array}{c}\text { Orbital } \\
\text { Speed } \\
(\mathrm{km} / \mathrm{h})\end{array}$ \\
\hline \multirow{2}{*}{ 1) Mars } & \multirow{2}{*}{0.642} & \multirow{2}{*}{867} & Phobos & 9.38 & 10.6 & 7695 \\
\hline & & & Deimos & 23.46 & 2.4 & 4868 \\
\hline \multirow[t]{3}{*}{ 2) Earth } & 5.97 & 1677 & Moon & 384.4 & 0.073 & 3679 \\
\hline & & & Miranda & 129.9 & 0.66 & 23,923 \\
\hline & & & Ariel & 190.9 & 13.5 & 19,844 \\
\hline \multirow[t]{5}{*}{ 3) Uranus } & 86.8 & 9310 & Umbriel & 557 & 11.7 & 16,821 \\
\hline & & & Titania & 436 & 35.2 & 13,110 \\
\hline & & & Oberon & 584 & 30.1 & 11,320 \\
\hline & & & Naiad & 23.2 & 0.002 & 43,350 \\
\hline & & & Thalassa & 25.2 & 0.004 & 42,129 \\
\hline \multirow[t]{3}{*}{ 4) Neptune } & 102 & 10,231 & Despina & 27.7 & 0.02 & 41,045 \\
\hline & & & Galatea & 37.2 & 0.04 & 37,836 \\
\hline & & & Larissa & 48.8 & 0.05 & 35,238 \\
\hline
\end{tabular}




\section{Continued}

\begin{tabular}{|c|c|c|c|c|c|c|}
\hline \multirow{5}{*}{ 5) Saturn } & \multirow{5}{*}{568} & \multirow{5}{*}{17,775} & Mimas & 185.5 & 0.379 & 51,684 \\
\hline & & & Enceladus & 238 & 1.08 & 45,471 \\
\hline & & & Tethys & 294.7 & 6.18 & 40,879 \\
\hline & & & Dione & 377.4 & 11.0 & 36,036 \\
\hline & & & Rhea & 527 & 23.1 & 30,531 \\
\hline \multirow{4}{*}{ 6) Jupiter } & \multirow{4}{*}{1899} & \multirow{4}{*}{45,255} & Io & 421.6 & 893.2 & 62,382 \\
\hline & & & Europa & 670.9 & 480 & 49,613 \\
\hline & & & Ganymede & 1070 & 1481.9 & 39,103 \\
\hline & & & Calisto & 1883 & 1075.9 & 29,531 \\
\hline
\end{tabular}

Data presented in this table were adapted from https://nssdc.gsfc.nasa.gov/planetary/factsheet/ and related pages. Only for the moon was actual value derived from the NASA's website; all other values were calculated from the values for the orbital parameters posted at the website. For calculating the orbits of the small satellites, where only semi-major axes were provided, they were used; since all satellites' values were thus affected, we accepted that limitation. ${ }^{*}$ The masses for all of the planets and earth's moon were $\times 10^{24} \mathrm{~kg}$ and for the satellites of Mars were $\times 10^{15} \mathrm{~kg}$; for Jupiter's moons were $\times 10^{21} \mathrm{~kg}$; for Saturn's, Uranus' and Neptune's were $\times 10^{20} \mathrm{~kg}$.

Table 2(b). Planetary axial rotation vs. satellites' axial rotation speed (Synchronously rotating satellites).

\begin{tabular}{|c|c|c|c|c|c|c|}
\hline \multicolumn{3}{|c|}{ Planets } & \multicolumn{4}{|c|}{ Satellites } \\
\hline & $\begin{array}{c}\text { Mass } \\
\left(10^{24} \mathrm{~kg}\right)\end{array}$ & $\begin{array}{l}\text { Axial } \\
\text { Rot. } \\
\text { Speed } \\
(\mathrm{km} / \mathrm{h})\end{array}$ & & Mass $^{*}$ & $\begin{array}{l}\text { Dist. } \\
\text { from } \\
\text { Mother } \\
\left(10^{3} \mathrm{~km}\right)\end{array}$ & $\begin{array}{c}\text { Axial. } \\
\text { Rot. } \\
\text { Speed } \\
(\mathrm{km} / \mathrm{h})\end{array}$ \\
\hline \multirow{2}{*}{ Mars } & \multirow{2}{*}{0.642} & \multirow{2}{*}{867} & Phobos & 10.6 & 9.38 & 9.33 \\
\hline & & & Deimos & 2.4 & 23.46 & 1.25 \\
\hline Earth & 5.97 & 1677 & Moon & 0.073 & 384.4 & 16.7 \\
\hline \multirow{5}{*}{ Uranus } & \multirow{5}{*}{86.8} & \multirow{5}{*}{9130} & Miranda & 0.66 & 129.9 & 44 \\
\hline & & & Ariel & 13.5 & 190.9 & 60.7 \\
\hline & & & Umbriel & 11.7 & 557 & 38 \\
\hline & & & Titania & 35.2 & 436 & 23.7 \\
\hline & & & Oberon & 30.1 & 584 & 14.8 \\
\hline \multirow{5}{*}{ Neptune } & \multirow{5}{*}{102} & \multirow{5}{*}{10,231} & Naiad & 0.002 & 23.2 & 31.5 \\
\hline & & & Thalassia & 0.004 & 25.2 & 36.9 \\
\hline & & & Despina & 0.02 & 27.7 & 60.5 \\
\hline & & & Galatia & 0.04 & 37.2 & 54.3 \\
\hline & & & Larissa & 0.05 & 48.8 & 47 \\
\hline \multirow{5}{*}{ Saturn } & \multirow{5}{*}{568} & \multirow{5}{*}{17,775} & Mimas & 0.379 & 185.5 & 51.6 \\
\hline & & & Enceladus & 1.08 & 238 & 44.5 \\
\hline & & & Tethys & 6.18 & 294.7 & 40.8 \\
\hline & & & Dione & 11.0 & 377.4 & 36 \\
\hline & & & Rhea & 23.1 & 527 & 30.7 \\
\hline \multirow{4}{*}{ Jupiter } & \multirow{4}{*}{1899} & \multirow{4}{*}{42,255} & Io & 893.2 & 421.6 & 269.6 \\
\hline & & & Europa & 480 & 670.9 & 115.2 \\
\hline & & & Ganymede & 1481.9 & 1070 & 95.7 \\
\hline & & & Callisto & 1075.9 & 1883 & 37.8 \\
\hline
\end{tabular}

Data in this table were adapted from https://nssdc.gsfc.nasa.gov/planetary/factsheet/ and related pages. Only for the moon was actual values derived from NASA's website; all other values were calculated from the values for the orbital parameters posted on that site. For calculating the axial rotation speeds, either using the "median axis radius" given by NASA, or by calculating it from the data provided (for the small satellites, where their shapes are not spherical) were used to determine the circumference. Since the satellites were synchronously rotating, for axial rotation period, the orbital period was used. Then, the satellites' orbital rotation was calculated from the two values. ${ }^{*}$ The masses for satellites of Mars were $\times 10^{15} \mathrm{~kg}$; for moon it was $\times 10^{24} \mathrm{~kg}$, for Uranus', Neptune's and Saturn's were $\times 10^{20} \mathrm{~kg}$; for Jupiter's they were $\times 10^{21} \mathrm{~kg}$. 
In this Table we compare the axial rotation speeds of the planets with the orbital speeds of their respective most proximal, synchronously rotating satellites. Included in the analyses are Mars and its two moons, the earth and its moon and the gas/ice giants with their closest 4 or 5 largest moons. It shows a close relationship between the two; the faster the axial rotation of the mother body, the faster the satellites orbiting it. The only deviation was between the satellites of Mars and earth; the former display faster orbits than the latter, although earth's axial rotation is faster than that of Mars; we suspect this to be due to the almost 15 - 30 times larger distances between our moon and the earth, compared to the moons of Mars. However, the orbital speeds of these three moons are considerably slower than those of the moons of the gas giants. Note that all the satellites represented in this figure are synchronously rotating. These findings confirm our conviction that spin of celestial bodies is purposeful and necessary to move satellites in orbit in the right direction and speed. It is as though the mother bodies grab the satellites and move them around in orbit in the same direction as their own axial rotation, thus determining also the orbital speed of the satellites. It is noteworthy that this relationship will at once also assure the counterclockwise direction of orbits of satellite bodies. This table also confirms the linear relationship between the masses of the bodies and their own axial rotation speeds. We can also see that, while orbiting in the speed rates appropriate for the mother bodies' axial rotation speeds, the farther away the synchronously rotating satellites are, correspondingly slower the satellites' orbital speeds.

When similar comparisons were made between the closest non-synchronously and negatively rotating satellites, they did not show the same close relationship with the mother body's axial rotation speeds. We think this finding attests to the rapidly diminishing gravitational/spin effect on the more peripheral bodies, and thus the mother body's diminishing ability to move the satellite bodies with fidelity, unlike on the synchronously rotating satellites.

Shown in this table are data pertaining to the masses and axial rotation speeds of the planets as those in Table 1 but, they are compared to the axial rotation rates of the satellites. Note the positive relationship between these parameters; the larger the mother body, and faster it rotates, the faster the axial rotation of the satellite bodies. Once again, we can conclude that the mother bodies control even the axial rotation rates of these satellites. Why this relationship is there, is not known but we speculated in a prior paper [1] that this may be to keep the axes of the satellites almost parallel to the mother's axis and thus assuring continuing confluent influence over the satellite bodies. Mention should be made of the cases where the autonomous axial rotation of planets tend to override the effect emanating from mother bodies, when the satellite bodies are large and situated far away from the mother. A good example is the case of the gas and ice giants, all of which rotate at much faster rates than what one would expect from the rotational influence from Sun, from such great distances. This is due to the fact that the same distance diminishes the rotational influence from Sun so much that the inherent axial rotation of the giant planets supersede. 
Table 3. Orbital parameters of satellites of Jupiter*.

\begin{tabular}{|c|c|c|c|c|c|}
\hline Satellites: & $\begin{array}{c}\text { Radius } \\
(\mathrm{km})\end{array}$ & $\begin{array}{c}\text { Distance from } \\
\text { Jupiter }^{\Delta} \\
\left(10^{3} \mathrm{~km}\right)\end{array}$ & $\begin{array}{l}\text { Orbital } \\
\text { Period } \\
\text { (Days) }\end{array}$ & $\begin{array}{c}\text { Rotation } \\
\text { Period } \\
\text { (Days) }\end{array}$ & $\begin{array}{c}\text { Inclination } \\
\text { (Degrees) }\end{array}$ \\
\hline \multicolumn{6}{|l|}{ A) Galilean: } \\
\hline Io & 1821.6 & 421.8 & 1.769138 & $S$ & 0.04 \\
\hline Europa & 1560.8 & 671.1 & 3.551181 & $S$ & 0.47 \\
\hline Ganymede & 2631.2 & 1070.4 & 7.154553 & $S$ & 0.18 \\
\hline Calisto & 2410.3 & 1882.7 & 16.689017 & S & 0.19 \\
\hline \multicolumn{6}{|l|}{ B) "Lesser" } \\
\hline Metis & $30 \times 20 \times 17$ & 128 & 0.294779 & $S$ & 0.06 \\
\hline Adrastea & $10 \times 8 \times 7$ & 129 & 0.298260 & S & 0.03 \\
\hline Amalthea & $125 \times 73 \times 64$ & 181.4 & 0.498179 & $\mathrm{~S}$ & 0.40 \\
\hline Thebe & $58 \times 49 \times 42$ & 221.9 & 0.6745 & S & 0.8 \\
\hline Themisto & 4 & 7507 & 132.02 & ND & 45.67 \\
\hline Leda & 5 & 11,170 & 240.92 & ND & 27.47 \\
\hline Himalia & 85 & 11,460 & 250.5662 & 0.4 & 27.63 \\
\hline Lysithea & 12 & 11,720 & 259.22 & ND & 27.35 \\
\hline Elara & 40 & 11,740 & 259.6528 & 0.5 & 24.77 \\
\hline S/2000 J11 & 2.0 & 12,560 & 287.0 & ND & 28.2 \\
\hline Carpo (S/2003 J20) & 3.0 & 16,990 & 456.1 & ND & 51.4 \\
\hline Euporie & 1 & 19,390 & $553.1 \mathrm{R}$ & ND & 147 \\
\hline Orthosie & 1 & 20,720 & $622.6 \mathrm{R}$ & ND & 145.9 \\
\hline Euanthe & 1.5 & 20,800 & $620.6 \mathrm{R}$ & ND & 148.9 \\
\hline Thyone & 2 & 20,940 & $627.3 \mathrm{R}$ & ND & 148.5 \\
\hline Mneme & 2 & 21,070 & $620.0 \mathrm{R}$ & ND & 148.6 \\
\hline Harpalyke & 2.2 & 21,110 & $623.3 \mathrm{R}$ & ND & 148.7 \\
\hline Hermippe & 2 & 21,130 & $633.9 \mathrm{R}$ & ND & 150.7 \\
\hline Praxidike & 3.4 & 21,150 & $625.3 \mathrm{R}$ & ND & 148.7 \\
\hline Thelxinoe & 2.0 & 21,160 & $628.1 \mathrm{R}$ & ND & 151.4 \\
\hline Helike & 4.0 & 21,260 & $634.8 \mathrm{R}$ & ND & 154.8 \\
\hline Iocaste & 2.6 & 21,270 & $631.5 \mathrm{R}$ & ND & 159.7 \\
\hline Ananke & 10 & 21,280 & $629.8 \mathrm{R}$ & ND & 148.9 \\
\hline Eurydome & 1.5 & 22,870 & $717.3 \mathrm{R}$ & ND & 150.3 \\
\hline Arche & 1.5 & 22,930 & $723.9 \mathrm{R}$ & ND & 165 \\
\hline Autonoe & 2 & 23,040 & $762.7 \mathrm{R}$ & ND & 152.9 \\
\hline Herse & 2 & 23,097 & $715.4 \mathrm{R}$ & ND & 164.2 \\
\hline Pasithee & 1 & 23,100 & $716.3 \mathrm{R}$ & ND & 165.4 \\
\hline Chaldene & 1.9 & 23,180 & $723.8 \mathrm{R}$ & ND & 165.4 \\
\hline Kale & 1 & 23,220 & $729.5 \mathrm{R}$ & ND & 165 \\
\hline
\end{tabular}


Continued

\begin{tabular}{cccccc}
\hline Isonoe & 1.9 & 23,220 & $725.5 \mathrm{R}$ & $\mathrm{ND}$ & 165 \\
Aitne & 1.5 & 23,230 & $730.2 \mathrm{R}$ & $\mathrm{ND}$ & 165.1 \\
Erinome & 1.6 & 23,280 & $728.3 \mathrm{R}$ & $\mathrm{ND}$ & 164.9 \\
Taygete & 2.5 & 23,360 & $732.2 \mathrm{R}$ & $\mathrm{ND}$ & 165.2 \\
Carme & 15 & 23,400 & $734.2 \mathrm{R}$ & $\mathrm{ND}$ & 164.9 \\
Sponde & 1 & 23,490 & $748.3 \mathrm{R}$ & $\mathrm{ND}$ & 151 \\
Kalyke & 2.6 & 23,580 & $743 \mathrm{R}$ & $\mathrm{ND}$ & 165.2 \\
Pasiphae & 18 & 23,620 & $743.6 \mathrm{R}$ & $\mathrm{ND}$ & 151.4 \\
Eukelade & 4 & 23,660 & $746.4 \mathrm{R}$ & $\mathrm{ND}$ & 165.5 \\
Megaclite & 2.7 & 23,810 & $752.8 \mathrm{R}$ & $\mathrm{ND}$ & 152.8 \\
Sinope & 14 & 23,940 & $758.9 \mathrm{R}$ & $\mathrm{ND}$ & 158.1 \\
Hegemono & 3 & 23,950 & $739.6 \mathrm{R}$ & $\mathrm{ND}$ & 155.2 \\
Aoede & 4 & 23,980 & $761.5 \mathrm{R}$ & $\mathrm{ND}$ & 158.3 \\
Kallichore & 2 & 24,040 & $764.7 \mathrm{R}$ & $\mathrm{ND}$ & 165.5 \\
Callirrhoe & 4 & 24,100 & $758.8 \mathrm{R}$ & $\mathrm{ND}$ & 147.1 \\
Cyllene & 2 & 24,350 & $737.8 \mathrm{R}$ & $\mathrm{ND}$ & 149.3 \\
Kore & 2 & 24,540 & $779.2 \mathrm{R}$ & $\mathrm{ND}$ & 152.4 \\
\hline
\end{tabular}

$\mathrm{C}=$ Newly discovered satellites S/2000 J2 to S/2011 J2 have orbital periods from 504 to 982.5 ; all exhibit reverse "motion" and orbital inclination from 140.8 to 165 . Numerous peripheral newly discovered unnamed satellites are not included in this Table. Most of them rotate negatively. $\mathrm{S}=$ Synchronous rotation (rotation period is the same as orbital period) $\mathrm{R}=$ Retrograde rotation ND $=$ No data available ${ }^{\Delta}$ Distance from Jupiter $\left(10^{3} \mathrm{~km}\right)=$ Semi-major Axis. ${ }^{*}$ Adapted from:

http://nssdc.gsfc.nasa.gov/planetary/factsheet/joviansatfact.html 16 July 2019 Reproduced with kind permission of Physics Essays Publication, http://physicsessays.org/ with modifications. Note the synchronous rotation in the closest moons, transitioning to nonsynchronous and then to negative rotations in distant satellites.

This table lists the satellites of the gas giant Jupiter, as a representative of all the gas/ice giants, all of which have multiple satellites. It clearly shows the progression of the orbital motions of the satellites from, "synchronous rotation" in the closest moons with no or very limited axial tilts (in the first seven satellites, Io to Thebe), to nonsynchronous rotation in the satellites that are farther away and with intermediate degrees of axial tilts, but less than $90^{\circ}$ (Next 7 satellites, Themisto to Carpo). Finally, all peripheral, small satellites display "negative rotation" (opposite to the normal, counterclockwise rotation) and have axial tilts over $-140^{\circ}$ (all peripheral satellites from Euporie onwards). The major proximal satellites of Saturn and Uranus also display similar axial rotational, orbital relationships with their mother bodies. Data on Neptune's satellites is unavailable. We have offered detailed explanations of the above phenomena in our prior paper [1] but suffice it to stress that this table teaches us the way in which spin and gravity interact in most planetary systems in our solar system.

Figure 2 \& Figure 3:

Our explanation of the origin of synchronous and negative rotations of the satellites of the gas and ice giants: Illustrated below is our understanding and explanations of how these exquisite axial rotational phenomena are produced. The legends accompanying the figures explain the principles involved adequately. 


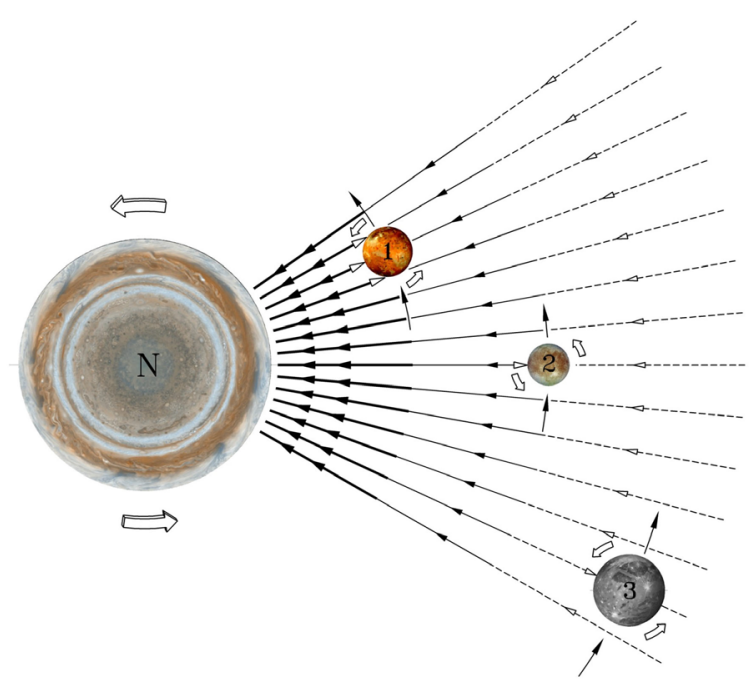

This schematic representation shows a view from above the North Pole of a Gas Giant and three "Synchronously rotating" major satellites. This diagram is not to scale. The gravitational pull of the Mother is represented by radiating lines, initially strongly solid and progressively thinning to represent progressive decrease in strength. All bodies are spinning on their axes in a counter-clockwise direction, as indicated by the open arrows surrounding them. Effect on the satellite \#1 is most intense, making it orbit the fastest, in a counter-clockwise direction around the Mother (solid arrow), and in the process encounters a strong drag on its leading edge. This directs the satellite to spin on its axis in a counter-clockwise fashion, thus augmenting its inherent tendency to spin in that same direction. The 2nd and 3 rd satellites encounter less intense gravitational pull and, although still spinning synchronously, both the orbital period and axial rotation period are greater.

Figure 2. A gas giant and three of its synchronously rotating major satellites. Reproduced from Applied Phys. Res. Vol 12, No 2, 2020 http://dx.dol.org/10.5539/apr.c12n2p.

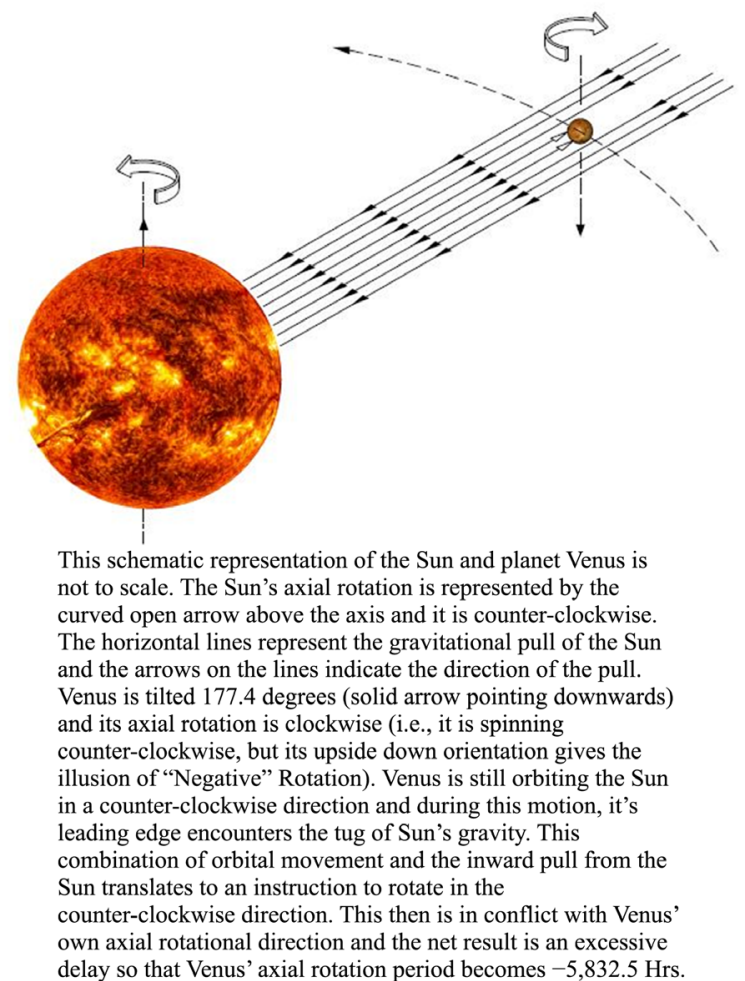

Figure 3. Mechanism underlying the "negative" rotation of the planet venus and the inordinate delay. Reproduced from Appl. Phys. Res. Vol 12, No 2, 2020; http://dx.dol.org/.10.5539/apr.v12n2p1. 


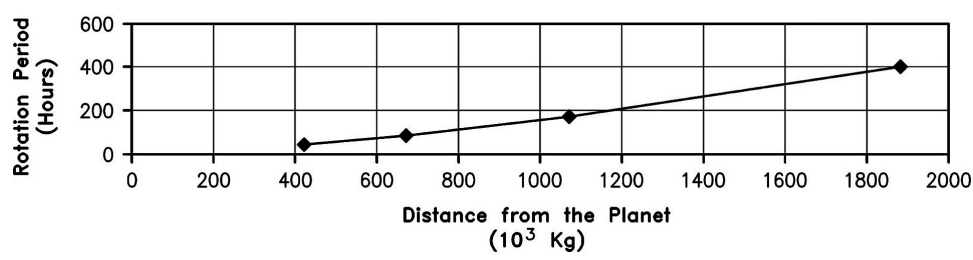

(a)

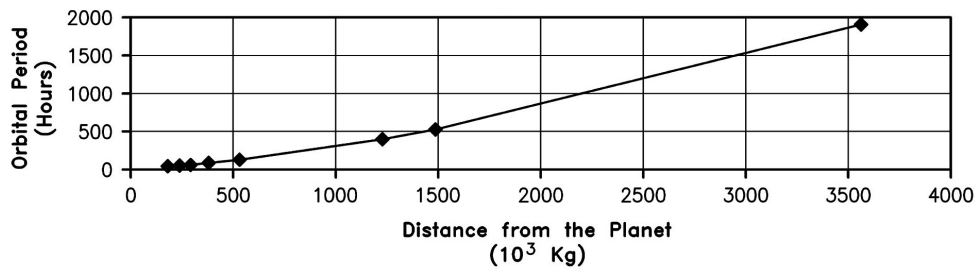

(b)

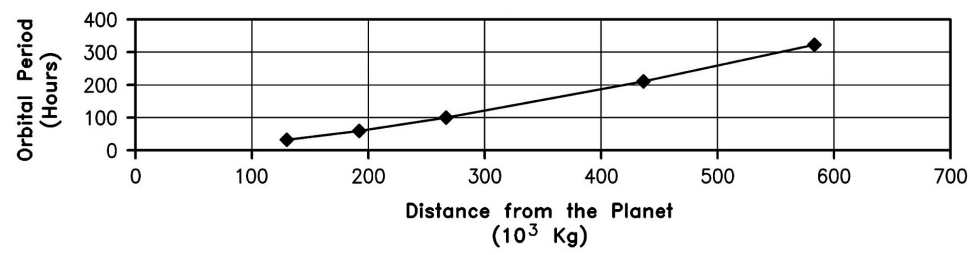

(c)

Figure 4. (a) Distance from the planet vs. rotation period of the moons for the major satellites of Jupiter; (b) Distance from the planet vs. orbital period of the moons for the major satellites of Saturn; (c) Distance from the planet vs. orbital period of the moons for the major satellites of Uranus. These figures compare the distance from the mother body vs. the rotation period (orbital period in Saturn and Uranus) of the synchronously rotating major satellites of Jupiter, Saturn and Uranus. All three comparions indicate an extremely positive linear relationship $(r=0.9959,0.9893$ and 0.9962$)$.

(Derived from Table 3 and Reproduced from [1]). As noted before, the figure lists major moons of two gas giants and an ice giant, all of which rotate synchronously. The main message to get from this figure is the increasing orbital period with distances from the mother bodies (an indirect measure of orbital speed). All the represented moons are rotating synchronously, with a good correlation between the distance from the mother and the satellites' orbital periods. This graph shows a linear relationship $(r=0.9959,0.9893$ and 0.9962 , respectively). Since the bodies are rotating synchronously, this also necessarily means that the axial rotation speeds of these satellites also have a positive relationship with the mother bodies' axial rotation speeds.

Table 4. Selected parameters of stars in sun's neighborhood.

\begin{tabular}{lccccc}
\hline \multicolumn{1}{c}{ Star } & $\begin{array}{c}\text { Distance } \\
\text { (Light Years) }\end{array}$ & Radius* & Mass $^{*}$ & $\begin{array}{c}\text { Rad. Vel } \\
\mathrm{km} / \mathrm{sec}\end{array}$ & $\begin{array}{c}\text { Rot. Vel } \\
\mathrm{km} / \mathrm{sec}\end{array}$ \\
\hline 1) Proxima Centauri & 4.24 & 0.154 & 0.122 & -22.20 & $<0.1$ \\
2) Alpha Centauri A & 4.37 & 1.22 & 1.1 & -21.4 & $2.7 \pm 0.7$ \\
3) Alpha Centauri B & 4.37 & 0.86 & 0.907 & -18.6 & $1.1 \pm 0.8$ \\
4) Barnaard's star & 5.96 & 0.196 & 0.144 & -110.6 & $<2.5$ \\
\hline
\end{tabular}




\section{Continued}

\begin{tabular}{|c|c|c|c|c|c|}
\hline 5) Wolf 359 & 7.86 & 0.16 & 0.09 & +19 & $<3.0$ \\
\hline 6) Sirius A & 8.6 & 1.71 & 2.063 & -5.5 & 16 \\
\hline 7) Luyten $726-8$ & 8.73 & 0.14 & 0.102 & +29 & 28.2 \\
\hline 8) Ross 154 & 9.6 & 0.24 & 0.17 & -10.7 & 3.5 \\
\hline 9) Ross 248 & 10.29 & 0.16 & 0.136 & -75.2 & 1.2 \\
\hline 10) Ross 128 & 11 & 0.197 & 0.168 & -31 & N/A \\
\hline 11) 61 Cygni A & 11.4 & 0.665 & 0.7 & -65.9 & N/A \\
\hline 12) 61 Cygni B & 11.4 & 0.595 & 0.63 & -64.4 & N/A \\
\hline 13) Procyon A & 11.46 & 2.05 & 1.50 & -3.2 & 3.16 \\
\hline 14) Epsilon Indi & 11.87 & 0.732 & 0.754 & -40.4 & 1.46 \\
\hline 15) Vega & 25 & $2.36 \times 2.82$ & 2.1 & -13.9 & 20.48 \\
\hline 16) Arcturus & 36.7 & 25.4 & 1.08 & -5.19 & 2.4 \\
\hline 17) Aldebaran & 65.3 & 44.13 & 1.16 & 54.26 & $3.5 \pm 1.5$ \\
\hline 18) Beta Carinae & 113.2 & 6.8 & 3.5 & -5.2 & 145.7 \\
\hline 19) Achernar & 139 & $7.3 \times 11.4$ & 6.7 & +16 & 250 \\
\hline 20) Alha Arae & $270 \pm 20$ & 4.5 & 9.6 & 0 & 375 \\
\hline 21) Canopus & 310 & 71 & 8 & +20.3 & 9 \\
\hline 22) Polaris & $323-433$ & 37.5 & 5.4 & -17 & 14 \\
\hline 23) Pleione & 392 & 3.2 & 3.4 & +4.4 & 329 \\
\hline 24) Epsilon Aurigae & $653-1500$ & $143-358$ & $2.2-15$ & 10.4 & 54 \\
\hline 25) PZ Cassiopeiae & 2810 & 1062 & N/A & -45.68 & 45 \\
\hline 26) Rho Cassiopeiae & $\sim 3400$ & $636-981$ & 40 & -47 & 25 \\
\hline 27) VY Canis Majoris & $\sim 3820$ & 1420 & 17 & 41 & 300 \\
\hline 28) KY Cygni & $\sim 3600$ & 672 & 25 & N/A & N/A \\
\hline 29) UY Scuti & $\sim 5100$ & 755 & $7-10$ & +18.33 & 18 \\
\hline 30) V382 Carinae & 5930 & 485 & 20 & +6 & N/A \\
\hline 31) V915 Scorpii & 5436 & 760 & N/A & +46 & N/A \\
\hline 32) Eta Carinae & 7500 & $\sim 240$ & $120-200$ & -25 & N/A \\
\hline 33) VFTS 102 & 164,000 & N/A & $\sim 25$ & +228 & $610 \pm 30$ \\
\hline
\end{tabular}

The data for this table were derived from published material online, mainly from Wikipedia.org but, some were confirmed or corrected by values posted in other sites, as well as from nasa.gov website. Radius and mass are expressed as multiples of solar radius or solar mass; N/A = Data not available.

This table compares the equatorial radii, masses, distances from earth, radial velocities (the stars' movement across the galaxy), and the speeds of the axial rotation of a selection of stars in the Milky Way Galaxy; the stars were randomly selected based mainly on their radii compared to those of our sun and listed in increasing order of distances from earth [5]. The only other consideration was the availability of essential data such as mass, radius, radial velocity and rota- 
tional velocity. Although there is a definite suggestion of increased axial rotation rates with the masses and radii, when both the radii and masses are similar, (stars $1-15,19$ and 23) it is not strictly linear. It is also noteworthy that both the availability of complete data and similar values of masses and radii are in stars that are the closest to the sun. This means the readings are more accurate and more available for close-by stars. When the radii and masses do not correspond, which also are in stars that are much farther away, the rotational values are quite unpredictable. Even so, one does notice larger rotational speeds in larger stars (stars 18, 19, 20,33). We infer from the above that the farther away the stars are from the observers, the less accurate the readings are. Therefore, only with more accurate readings in the future can we have conclusive evidence for the patterns of stars' behavior. In general, we believe, the data presented in this table does not refute our contention that the larger a star, faster it will rotate on its axis.

Table 5. Selected parameters of large galaxies.

\begin{tabular}{|c|c|c|c|c|c|c|}
\hline Name & $\begin{array}{c}\text { Distance } \\
\text { (LY) }\end{array}$ & Mass $^{*}$ & $\begin{array}{c}\text { Size } \\
\text { (Diam.) } \\
(\mathrm{LY})\end{array}$ & $\begin{array}{l}\text { No. of } \\
\text { Stars }\end{array}$ & $\begin{array}{c}\text { Helio-Radial } \\
\text { Vel }(\mathrm{km} / \mathrm{s})\end{array}$ & $\begin{array}{c}\text { Galacto- } \\
\text { Centric } \\
\text { Vel }(\mathrm{km} / \mathrm{s})\end{array}$ \\
\hline 1) $1 \mathrm{C} 1101$ & $1.045 \pm 0.073 \mathrm{~B}$ & N/A & $4 \mathrm{M}$ & $100 \mathrm{~T}\left(10^{14}\right)$ & $23,368 \pm 26$ & $23,395 \pm 26$ \\
\hline $\begin{array}{l}\text { 2) 3C } 348 \\
\text { (Hercules A) }\end{array}$ & $2.1 \mathrm{~B}$ & $1000^{*}$ & $1.5 \mathrm{M}$ & N/A & N/A & N/A \\
\hline 3) A2261-BCG & $3 \mathrm{~B}$ & $10^{*}$ & $1 \mathrm{M}$ & $10 \mathrm{~T}\left(10^{13}\right)$ & N/A & N/A \\
\hline 4) ESO 306-17 & $493 \mathrm{M}$ & $2.5 \mathrm{arc} . \mathrm{Sec}$ & $1 \mathrm{M}$ & N/A & N/A & N/A \\
\hline 5) UGC 2885 & $232 \mathrm{M}$ & $463 \mathrm{~K}$ ly & 800 & $1 \mathrm{~T}$ & N/A & N/A \\
\hline 6) Comet & $3.2 \mathrm{~B}$ & $3.8 \times 10^{8} \mathrm{M} \odot$ & $600 \mathrm{~K}$ & N/A & $3.4 \mathrm{M}$ & N/A \\
\hline $\begin{array}{l}\text { 7) NGC } 6872 \\
\text { (Condor Gal) }\end{array}$ & $212 \mathrm{M}$ & $>10^{11} \mathrm{M} \odot$ & $522 \mathrm{~K}$ & N/A & 4555 & 4443 \\
\hline 8) ESO 444-46 & $640 \mathrm{M}$ & $10,000^{*}$ & $402 \mathrm{~K}$ & N/A & 14,061 & $\mathrm{~N} / \mathrm{A}$ \\
\hline 9) Tadpole & $420 \mathrm{M}$ & N/A & $280 \mathrm{~K}$ & N/A & $\mathrm{N} / \mathrm{A}$ & N/A \\
\hline 10)Andromeda & $2.54 \mathrm{M}$ & $1.76^{\star}$ & $\sim 220 \mathrm{~K}$ & $1 \mathrm{~T}$ & -301 & -120 \\
\hline 11) Milky Way & & $1 \times 10^{12} \mathrm{M} \odot$ & 105.2 & $250-500$ & 210 & N/A \\
\hline
\end{tabular}

$\mathrm{LY}=$ Light years $\mathrm{K}=\times 1000 \mathrm{M}=$ Million $\mathrm{B}=$ Billion $\mathrm{T}=$ Trillion; $\mathrm{N} / \mathrm{A}=$ Data not available; ${ }^{\star}=\times$ Mass of Milky Way Galaxy; $\mathrm{M} \odot=\times$ Mass of Sun.

The data for this table were derived from our review of astronomy/astrophysical journals and various online sites, including nasa.gov, Wikipedia.org and others. There is great paucity of data for the parameters we were particularly interested in (axial rotation speeds and radial velocity, vs mass/size of the galaxies). We tried to select large galaxies and compare them with medium-sized ones such as our Milky Way Galaxy. Apparently, the largest of the galaxies are also the farthest and clearly the availability of data is severely hampered by that fact alone. Thus, on this table we are left with comparison of only a few galaxies (Nos.1, 6, 7,8 vs $10 \& 11$ ). Even with this sparse data, there is a good hint that the galac- 
to-radial and helio-radial velocities are higher, the larger the galaxy is. Taken together with the recent observation [6] of the "Super Spirals" rotating even faster, we can safely predict that future availability of accurate information will confirm our belief.

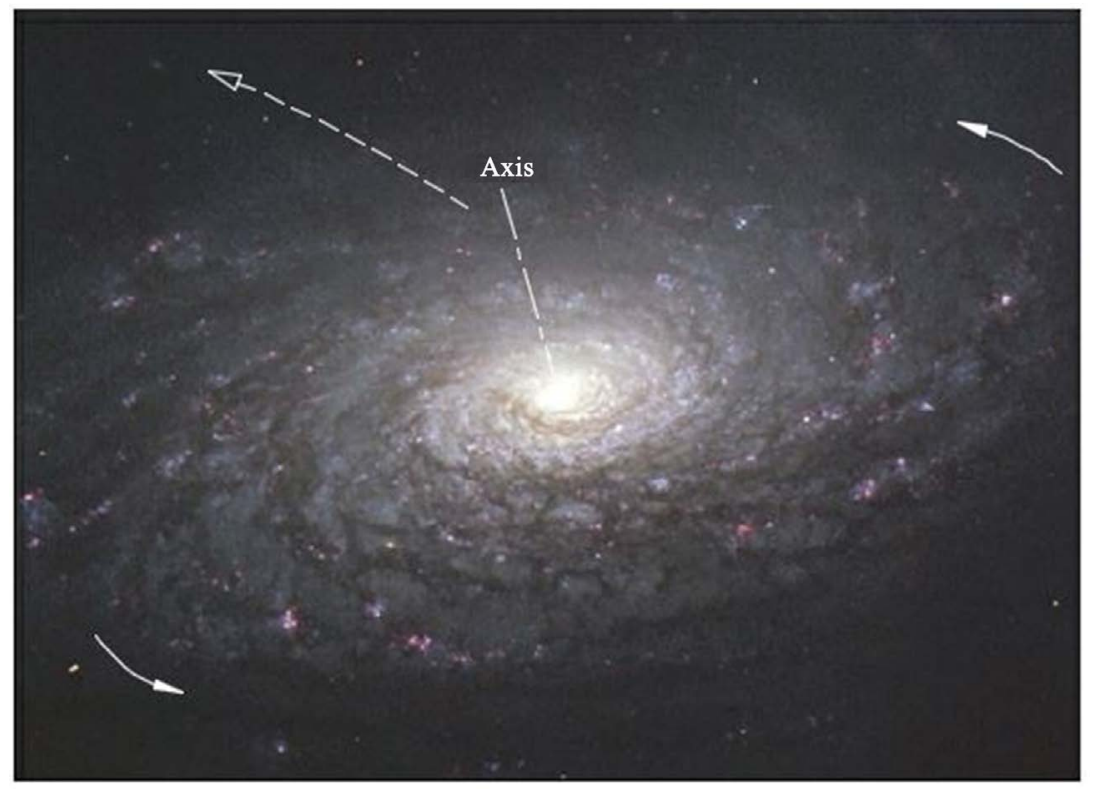

Figure 5. This image is adapted from: http://nasa.gov. This figure is an artist's rendition of a spiral galaxy from above and to the right of the galaxy. The two closed arrows on opposite sides of the galaxy (at 5 mins and around 40 mins) show a counterclockwise direction of axial rotation. The open arrow shows the direction taken by the galaxy as it moves through the universe (as opposed to its axial rotation). This movement is also in the counterclockwise direction through the universe, across the vastness of space.

\section{Discussion}

Aryabhata I, (476 - $550 \mathrm{CE})$ the great mathematician/astronomer of ancient India explained how the illusion of the "heavens rotating" around the earth was actually due to the earth rotating on its axis [7] [8] [9] [10]. While this is an early recognition of axial rotation, his view of the solar system described the known planets and the sun orbiting the earth. True heliocentric view of the solar system came much later. Although science had recognized the universally observed axial rotation and the orbital motions of celestial bodies, the connection between the two that we are making in this paper is novel. Newton's mutual gravitation by itself does not explain how the orbits occur; for explaining that, the current cosmology invokes his $1^{\text {st }}$ law of motion in conjunction with the gravitation. However, those combinations do not explain the strictly counterclockwise direction of the orbits in almost all of the astronomical systems; neither does it explain the axial rotation of bodies, or the ecliptic location of most bodies' orbits. This failure to recognize the true nature of the incessant axial rotational/orbital motions as being of importance in the makeup of the universe was probably due to the notion of "conservation of angular momentum' that is common in con- 
ventional scientific thinking. The error was in not recognizing the importance of this spin in celestial body motion mechanics, as well as the realization that this property is inherent in all congregations of matter. Our studies convince us that, rather than being a remnant of a prior astrophysical phenomenon, the spin/orbital combination is purposeful, as we will presently proceed to discuss below. In our prior articles dealing with the universal presence and importance of the axial rotation of celestial bodies, we have proposed that the mutual gravitation, combined with the axial spin of the mother bodies are truly behind the orbits of the satellite bodies and, in this process, the resultant centrifugal force is a necessary accomplice. In the following sections we will discuss our findings and offer our arguments to explain how these forces combine to promote orderly motions of bodies in the universe. We hope to propose this mechanism as an alternative to the Standard Model of Cosmology.

\section{Axial Spin Is a Fundamental Property of Matter}

All fundamental particles are known to spin on their axes; electrons both spin on their axes and orbit the nucleus of atoms. Even photons, one of the force-carrying elementary particles (bosons), are known to spin [10]-[20]; it is not known if gravitons, another force-carrying agent also display this property. All freestanding bodies, the planets and their satellites, as well as all stars are known to spin on their axes. The finding of a linear positive relationship between the mass of a planet to the speed of its axial rotation (Figure 1), is a good hint that the axial spin is an inherent, fundamental property of matter. A spectacular example of this intrinsic property displayed by matter is the case of the neutron stars. These remnants of large stars that underwent supernova explosion, after losing most of their mass and are left with almost exclusively neutrons, and are of roughly the size of medium-sized cities, spin on their axes at several times a second, up to 716 times a second! [21]. We explain this ability of neutron stars to rotate so rapidly as evidence of how fast the nuclei of atoms rotate on their axes; these remnants of stars, are just displaying this natural tendency, unfettered by friction, in the vacuum at the areas where they are situated, and behave like a nucleus of an atom. Also, the finding that even planets and satellites that are upside down in orientation, still rotate on their axes in the appropriate way, that is, counterclockwise, is telling.

Stars and most of the congregations of stars, the galaxies also spin on their axes [22] [23] and their speed of axial rotation also seem to depend on the size of the body (ies) [6]. Table 4 addresses the radial motion as well as the axial rotation rates of several stars and they were compared to their radii and masses. While the data is quite incomplete, when they are available and both the stars' radii and masses are comparable, there is a good hint that the stars also rotate on their axes faster, the larger they are. It is less certain that they also move in space in the galaxies, laterally, also faster the larger they are. Similarly, in the case of galaxies, the data are even more sparse. However, here also, there is a good hint 
that larger galaxies rotate on their axes faster than the smaller ones. Again, the lateral motion of galaxies is not available in sufficient details to make any conclusions. Overall, even with the paucity of data that is available in the literature, a general tendency towards faster rotation and lateral movement in larger galaxies is observable. It is not difficult to draw the conclusion from all of the above that, this ubiquitous finding, axial spin of congregations of matter, is a fundamental property of matter and it has the important function of both generating and maintaining celestial body motions.

Axial Spin of Celestial Bodies and the Direction of Orbits of Their Satellites Are in the Counterclockwise Direction:

All congregations of matter spin in a counterclockwise direction and this motion is continued in the orbital movements of all satellites around their respective mother bodies (with the sole exception of Triton, a major moon of Neptune, which orbits in the wrong direction) and in all planets and all the other major bodies in our solar system around the sun. As we noted above in Table 3 dealing with Jupiter and its satellites, and in our paper dealing with synchronous, nonsynchronous and negative rotations [1], all celestial movements are controlled by this arrangement. We have not presented in this paper the corresponding details about the satellites of Saturn and Uranus, for the sake of brevity but the closest moons of both of these planets also display synchronous rotation, while the satellites that are farther away either have nonsynchronous or reverse rotations, depending on the distance from the mother, just like in the case of Jupiter's satellites. There are not enough data available on Neptune's satellites to include in our report. These orderly orbital motions of the satellites teach us how the mother bodies are able to carry their satellites in exact orbits, at the necessary speeds, only in one direction, and thus avoid constant chaos. This direction of axial rotation is continued in the congregations of stars that form the galaxies. As expected, the direction of this rotation is also counterclockwise in spiral and elliptical galaxies [22] [23].

Mother Bodies' Axial Rotation Guides and Controls the Orbital Motion of the Satellites:

We have shown how the combined forces of axial spin and gravity determine the direction and speed of orbits of planets and their satellites; Table 2(a) clearly shows how the speed of axial rotation of the mother bodies determine the speed of orbits of the satellites. Thus, this effect is purposeful and is in exact proportions. We have also shown how the speed of axial rotation of the satellites is also influenced by the mother bodies [1], as Table 2(b) clearly shows. The predictable sequence of synchronous rotations in the closest satellites, followed by nonsynchronous and then negative rotations as shown in Table 3, bear witness to mother bodies' commanding influence on their satellites. No random occurrence of orbitals will explain these orderly transitions. It is not difficult to infer from the above observations that the mother body is using the combination of gravity and spin in charting the course of its satellites. Such influence clearly 
guides all planetary/satellite bodies' motions to proceed in the same direction.

The Other Solar System Bodies Also Align Themselves in the Equatorial Regions and Orbit the Sun (Or Planets, In the Case of the Rings), in the Counterclockwise Direction:

The solar system is home to trillions of smaller bodies that range in sizes from micrometers to several kilometers and come in many shapes. These organize themselves around the neighboring larger bodies and form their rings, or remain in distinct areas in the "asteroid belt" or in the "Kuiper belt". All of these bodies also exhibit one common feature; that is their ability to orbit the local planet or the sun in the counterclockwise direction, while remaining roughly along the ecliptic. A classic example of this organization is the rings of Uranus; while the planet is tilted on its axis over 90 degrees, the rings remain in the ecliptic location around the planet. The take away message from all these bodies' behavior is that regardless of how they were formed or what they are composed of, all bodies behave similarly when they are under the influence of larger celestial bodies, in orbit in the counterclockwise direction and along the ecliptic, and in defined speeds of orbit.

\section{The Solar Nebula and Exoplanets:}

When large clouds of matter destined to form a star and its system of planets and other bodies condense into a proto-planetary disk, it is already rotating. This rotation is also in the counterclockwise direction; conventional explanation of this rotation is that when the matter condenses, this direction of rotation is by chance and then it is continued in all the bodies involved, in a "conservation of angular momentum". We question this assumption, even at a statistical sense, as half of the solar nebulae should rotate in the clockwise direction, if that were by chance only. By our theory, it is very easy to explain this phenomenon; all congregations of matter will acquire the ability to spin and the larger bodies will take the smaller bodies in their gravitational influence around in orbits. We explain later in this Discussion, how such motions then become perpetual. Over the past twenty years "exo-planets" (planets orbiting other stars) have been discovered at increasing rates; as of April 2, 2020, this count was 4241 [24] [25] [26]. While scientists suspect these planets might also orbit their stars in the counterclockwise direction, direct observation of that has not been forthcoming. We hope to see new techniques that will help us determine that and also check the direction of rotation of the planets on their axes.

\section{The Other Ways in Which Spin and Gravity Interact:}

Our prior studies clearly showed that this ubiquitous property of matter is purposeful in many ways, in both maintaining the architecture and functioning of the universe. It is common knowledge that the orbital movements of electrons lead to the generation of magnetic moments; it emphasizes the fact that even at the level of fundamental particles [11] [12], the same arrangement exists. We proposed that the generation of magnetism in planets is also due to this same interaction [1]. Briefly, this process involves the molten iron in the interior of the earth, which is not rotating, but the rest of the planet's mass is rotating 
around it and this rotating mass generates the magnetism. We believe this magnetism is beneficial in ways other than by protecting the earth from the destructive power of solar flares; one such purpose is to help maintain the proper orientation of the planets, as well as (by the repulsion effect of like-poles) to help keep the planets at appropriate distances. The increase in the speed of axial rotation of planets depending on their masses (Figure 1) implies that it is meant to counteract the inherently increased gravitational effect of the larger masses of planets. Thus, a balance is struck. The increased gravity, along with the increase in axial rotation, which leads to increase in centrifugal force in exact amounts mean there remains an exact counterbalance in celestial motion mechanics. A simple extrapolation of such cooperation between axial spin, gravity and centrifugal force to the realm of the galaxies will help explain the motion of stars within them, as well as the motion of the whole galaxies across the universe. The reason for the inversion of the most peripheral satellites of the gas and ice giants to such an extent that they uniformly rotate negatively is not known. However, we speculate that this may be due to an "inversion" of the magnetic polarity in the farthest reaches of each planet's field of influence. This can be tested by experimentation with the satellites of the gas giants.

Another important function of the interaction of axial spin and gravity is to maintain the nearly spherical shape of stars, planets and other bodies of substantial size in the solar system. This brings us to the question why the spherical shape is essential or is preferred over other shapes for celestial bodies. We think spherical or nearly spherical shape makes the axial rotation of bodies easier than an irregular shape or even another geometrically symmetrical shape such as a cube, as these are likely significantly less efficient. It may even be the case that spheres would travel in space, as is the case with orbits, much easier than almost any other shape of bodies, as well, especially since they are also rotating on their axes.

Finally, some questions that readers and scientists might entertain about the subject matter that we are discussing. One question is, why the solar system does not have a progression from synchronously rotating large planets close to the sun, to nonsynchronous motion of the planets in the intermediate distances and negative or reverse rotation in all peripheral planets. In fact, both Mercury and Venus, the two closest planets to the sun are candidates for rotating synchronously. However, both of them do not rotate faster from being close to the sun; in fact, they rotate extremely slowly. This is despite the fact that they observe the inverse square law in their orbital speeds. We believe Mercury's odd behavior can be ascribed to the quantity of iron in its interior, and thus it behaves like a bar magnet standing next to a large, intensely magnetic body that the sun is. This effect from Sun is probably responsible for the extreme slowness of Mercury's axial rotation. In the case of Venus, which is tilted -174 degrees, its own inherent axial rotation is slowed down by the mechanisms we described in our paper [1]. We believe that because of these two oddballs being situated at such strategic locations close to the sun, the scientists were not able to appreciate 
synchronous rotation progressing to nonsynchronous rotations in the proximal planets in our solar system. We firmly believe that those star systems that do not have such unusual bodies situated next to the star, will display the expected pattern. In fact, some exo-planets that have been shown to be located close to and orbit at enormous speeds around their parent star may actually be cases of synchronously rotating planets [24] [25] [26].

While it is easy to explain why the gas/ice giants rotate on their axes extremely fast, (Jupiter rotates in $9.9 \mathrm{~h}$ Saturn in $10.7 \mathrm{~h}$ and Neptune in $16.1 \mathrm{~h}$ ); Uranus, which is tilted about 90 degrees and negatively rotating, still manages to rotate fast. How? We think part of the answer is that for axial rotation speed to diminish due to reverse rotation, the axial tilt has to exceed 120 degrees or so. Thus, Uranus manages to exhibit its natural speed of axial rotation commensurate with its mass and maintains a rotational speed of once in 17.2 hours.

The rest of the planets in general display nonsynchronous rotation. It will be interesting to see if the most peripheral small (“dwarf") planets have increased axial tilts and are rotating negatively, just like Pluto. It will also be of interest to check other star systems and see if the "exo-planets" follow the general pattern that we report in our own gas giants and their satellites. Across the universe there will be very many unusual findings. There may also be many star systems that have solid (terrestrial) planets without unusual features. Also, gaseous planets may abound in the universe and they also may rotate on their axes more rapidly, and independent of the central star.

How Are Large Bodies Influenced by the Mother Bodies to Orbit, and How Do Huge Congregations of Stars Move in Space?

It is appropriate to consider the question of how stars and other mother bodies influence satellite bodies, using mutual gravitation and spin, from vast distances. Gravity is a rather feeble force and it diminishes at geometric scale with distance (the inverse square law). We believe that this sequence of motion mechanics is accomplished because all bodies, where they are situated in deep space, are almost weightless. This is how a star like our Sun is able to move a rather large planet like Jupiter, from so far away. The same logic can be applied to explain how the axial rotation of large congregations of stars, the galaxies, occur. Since even the galaxies are essentially weightless, it would not require as much power to move the galaxy in a circular direction, unlike what has been proposed by scientists. The current teaching is to attribute the task of rotating the galaxy to a central "supermassive black hole" and its strength is determined mathematically. We instead suspect that the huge fireball in the center of the galaxy itself behaves like a huge star, except that it has the mass of billions of stars; it spins on its axis as expected and takes the rest of the galaxy with it.

We have an explanation for the peculiarity with the speed of motion of the stars in spiral galaxies. The finding that baffles scientists is that all stars in spiral galaxies, those closest to the center and the most peripheral ones, seem to move at about the same speed, quite unlike the orbital movements of planets and satellites in the solar system. We believe this is due to the fact that the flattened ga- 
laxy has stacks and stacks of stars, with of course their intense gravity such that, the whole galaxy behaves like a table top spinning on a central fulcrum; this motion is quite unlike the motion in our solar system, where the farthest planets orbit slower and cover longer distances. We think this is where the scientists went wrong, in trying to explain the stars' motion by using the laws that govern the orbits of the planets in this solar system. In the spiral galaxies, although all the stars are moving at about the same speed, the most peripheral stars take longer to cover the vast distances involved, compared to those stars situated near the center of the galaxies.

The axial rotation speed of spiral galaxies will yield another proof for our contention that this property is inherent and fundamental to all congregations of matter [27]-[34]. Like the axial rotation speed of the regular planets of our solar system, where the larger the planet, the faster its rotation, the spiral galaxies also likely display the same characteristic. Our Milky Way Galaxy, which is a medium-sized galaxy, 100,000 light years across, rotates at the rate of $130 \mathrm{miles} / \mathrm{sec}$ $(210 \mathrm{~km} / \mathrm{sec})$. In contrast, some "super spirals" (some as large as 450,000 light years across), are known to rotate at up to $350 \mathrm{miles} / \mathrm{sec}(579 \mathrm{~km} / \mathrm{sec})$ [5]. The explanation for this phenomenon in current teaching is to invoke the usual "default" idea of the presence of "incredibly large clouds or haloes of dark matter" [35]. The suggestion here is that this invisible and un-provable dark matter imparts so much extra gravity that somehow it translates to increased speed of axial rotation of galaxies. We fail to understand how having more gravity will impart this increased ability to rotate. On the other hand, by our idea of larger congregations of matter having the ability to rotate faster on their axes, it is easy to explain this phenomenon. Thus, the larger the congregation, the faster the spin; even at the level of stars and galaxies, the same law is in play.

The flaring outward of the arms of the spiral galaxies can be explained by the peripheral congregations of stars lagging behind as the whole galaxy is spinning in one direction, due to the distance traversed, in conjunction with the centrifugal force experienced by these arms in the process. The very same counterclockwise motion of the disk of the whole galaxy will tend to propel the galaxy itself in the direction of the open ends of the arms (see Figure 5); this makes the motion of the galaxy to be also counterclockwise, as reported by Longo [36]. Another phenomenon found in spiral galaxies is the not-so-rare collisions between galaxies. In the standard model of cosmology, a necessary consequence of "Big Bang" and the expanding universe, is a propulsion of all galaxies away from one another, apparently at close to the speed of light. It is thus inconceivable how then these galaxies that are flying apart from one another will bump into other galaxies. In our model, with the axial rotation speed and the lateral motion of galaxies depending on the size of the unit, one can easily see how, even though all of them are moving in the same counterclockwise direction, the larger galaxies could overtake the smaller, slower moving galaxies and collide with them. A good example of this process is the case of Andromeda Galaxy, which is approaching our Milky Way Galaxy, and scientists are predicting the two galaxies' 
collision/merger in the future.

Finally, we want to draw the distinction between our concept of spinning universe to what some investigators have proposed [37]. In their concept the galaxies and stars and all the lesser bodies acquire the ability to spin on their axes as a result of an explosive birth of the universe, akin to Big Bang, but apparently in a rotational event. Then, the theory goes, that spinning ability is carried on forever. This idea is thus an amalgam of the Big Bang and the "conservation of angular momentum". The real problem with this birth of the universe in a spinning model is that they are taking the concept of the conservation from just the solar nebula to all the way through the universe. We strongly believe that, just like all radiations, exemplified by light, when they (any body or radiation) travel close to large bodies with intense gravitation, the light rays are bent, and the bodies are pulled in. This also means that the transit of the rays and bodies is slowed; such encounters for billions of years will slow down all kinds of motions and eventually bring them to a stop. In our idea, the spin is an inherent ability of matter, and thus it remains independent of all sorts of influences and, given the right conditions, such as a frictionless state, the low gravitational distractions and perhaps the intense cold, this ability to spin is maintained perpetually. The beauty of this theory is that all the elements of this balanced interactions are readily observable and does not require invoking any esoteric force(s) such as dark matter, dark energy, negative energy etc.

How does one confirm this important role of the pervasive axial spin in the celestial body motion mechanics? We offer two different ways of obtaining supporting data. The first is doing exhaustive studies of the galaxies, to determine which way they turn on their axes ("handed-ness"). The study reported by Longo is a beginning in this search. However, the crucial data we need is which way the galaxies actually move in space; we believe such studies will prove that they actually move tangentially and not radially outward, as proposed by current cosmology. The second is an experiment designed to test our notion that all freestanding bodies in deep space, where the right conditions (perfect vacuum, almost no local gravitational influences and extreme cold) exist, will exhibit their inherent tendency to spin on their axes and orbit a local larger body. We propose taking several spherical objects made of different solid materials, with a bar magnet in each (to align the balls in the right polarity with the local celestial body) into deep space and leaving them there. Of course, they will be weightless and float freely. We predict that, given time, these bodies will spontaneously rotate on their axes, in the counterclockwise direction, and to orbit the local gravitationally active body, such as a planet or satellite or even a large asteroid.

\section{Conclusion}

Crucial to understanding the celestial motion mechanics as described in this paper, is recognition of the intrinsic beauty and functionality of the pervasive property of matter to spin; this is exemplified by the linear relationship between mass and speed of rotation of bodies. For example, the major satellites of the 
major planets in the solar system rotate on their axes at meager 9.33 to 269.6 $\mathrm{km} /$ hour, while their respective mother planets rotate between 867 and 45,255 $\mathbf{k m} /$ hour (see Table 2(b)). The stars on the other hand rotate at astonishing speeds between 0.1 and $610 \mathrm{~km} /$ second (see Table 4) and the galaxies rotate on their axes at even higher rates between 210 and 23,368 $\mathrm{km} /$ second (see Table 5). In all of these instances, the determining variable is the mass of the body; the larger the body, the faster its speed of axial rotation. However, there is another concept that is crucial to understanding our hypothesis. Here, one needs to free oneself from the constraints imposed by our earthbound existence and imagine the milieu that the celestial bodies are in; this includes the galaxies as well. In the vast void of space, where there is an almost perfect vacuum means there is no friction. Also, as the galaxies are separated by vast spaces, gravitational effects are minimal; this necessarily means that all bodies, regardless of their masses, are essentially weightless. What effect the almost absolute zero temperature that also exists in such deep space has on the celestial bodies, is unknown. However, superconductivity and altered forms of matter such as Bose-Einstein Condensate [38] [39] are consequences of such intense cold temperatures. Therefore, this is an area that needs to be investigated. Regardless the other extremes of conditions, just by the confluence of the vacuum and the weightlessness of bodies, even the galaxies, with their cargo of matter in all shapes and sizes, are still essentially like fluff, floating in space. Thus, their own axial rotation does not require as much energy as one might suspect. Further, this rotation of the galaxies on their own axes imparts a tangential motion to the galaxy, in the direction of the spin and they move in the same counterclockwise direction. We are tempted to compare such motion of the galaxies to that of Frisbees, which also tend to move in a curvilinear direction, once launched. The larger galaxies will move faster and then tend to bump into smaller, slower-moving galaxies; however, most of the time the motion of galaxies in the counter-clockwise direction means avoidance of collisions. This concept eliminates the need for proposing esoteric, unproven and un-provable ideas such as dark matter, dark energy, negative energy, dark haloes and so on, to explain the problems presented by current cosmological teaching. In doing so, the current teaching in science ignored the axial spin, the only readily observable, ubiquitous property of matter!

\section{Acknowledgements}

We wish to thank Ms. Rosie Gonzales for her excellent secretarial assistance. Equally important contributions were made by Mr. Jeff Nichols in preparing all the figures presented in this paper. We are also deeply indebted to Dr. Lloyd Taylor, who provided the statistical analyses and the original Figures 4(a)-(c). Our deep gratitude is due also to Dr. John Worrell for his probing questioning, various suggestions and his invaluable advices during the preparation of this and our prior articles. Finally, we and the scientific world owe incalculable gratitude to all the scientists in NASA and various other agencies, as well as all astronomy 
departments of universities across the world, who have spent countless hours of difficult observations, then cataloging and reporting the data on celestial bodies, without which our work and production of our manuscripts would not have been possible.

\section{Conflicts of Interest}

The author declares no conflicts of interest regarding the publication of this paper.

\section{References}

[1] Raghuprasad, P.K. (2020) Synchronous, Nonsynchronous and Negative Rotations: How Spin and Gravity Orchestrate Planetary Motions. Applied Physics Research, 12, 1-11. https://doi.org/10.5539/apr.v12n2p1

[2] Raghuprasad, P.K. (2020) Spin: Ubiquitous, Fundamental, Purposeful: Its Complementary Interactions with Gravity. Applied Science and Innovative Research, 4. http://www.scholink.org/ojs/index.php/asir https://doi.org/10.22158/asir.v4n3p1

[3] Banerdt, W.B., et al. (2020) Initial Results from the InSight Mission on Mars. Nature Geoscience, 13, 183-189.

[4] Johnson, C., et al. (2020) Crustal and Time-Varying Magnetic Fields at the InSight Landing Site on Mars. Nature Geoscience, 13, 199-204. https://doi.org/10.1038/s41561-020-0537-x

[5] Each Star Searched under Its Name. https://en.wikipedia.org

[6] Ogle, P.M., Jarrett, T., Lanz, L., Cluver, M., Alatalo, K., Appleton, P.N. and Mazzarella, J.M. (2019) A Break in Spiral Galaxy Scaling Relations at the Upper Limit of Galaxy Mass. The Astrophysical Journal Letters, 884, L11. https://doi.org/10.3847/2041-8213/ab459e

[7] Pearce, I.G. Indian Mathematics-Redressing the Balance. The Classical Period: II Aryabhata and His Commentator. https://mathshistory.st-andrews.ac.uk/Projects/Pearce/chapter-10/

[8] Hayashi, T. (1997) Aryabhata's Rule and Table for Sine-Differences. Historia Mathematics, 24, 396-406. https://doi.org/10.1006/hmat.1997.2160

[9] Kak, S. (2006) Aryabhata's Mathematics. RSA Conference, San Jose, 13-17 February 2006.

https://www.researchgate.net/publication/45901748_Aryabhata's_Mathematics

[10] http://en.wikipedia.org/wiki/Aryabhata

[11] Feynman, R.P. and Weinberg, S. (1987) Elementary Particles and the Laws of Physics: The 1986 Dirac Memorial Lectures. Cambridge University Press, Cambridge. https://doi.org/10.1017/CBO9781107590076

[12] Hofmeister, A. and Criss, R.E. Origin of Axial Spin and Orbital Rotation of the Solar System. https://ui.adsabs.harvard.edu/abs/2011AGUFM.P11A1587H/abstract

[13] Bouchard, F., Leon, I.D., Schulz, S.A., Upham, J., Karimi, E. and Boyd, R.W. (2014) Optical Spin-to-Orbital Angular Momentum Conversion in Ultra-Thin Metasurfaces with Arbitrary Topological Charge. Applied Physics Letters, 105, Article ID: 101905. https://doi.org/10.1063/1.4895620

[14] Zang, W, Qi, Q., Zhou, J. and Chen, L. (2014) Mimicking Faraday Rotation to Sort Orbital Angular Momentum of Light. Applied Physics Letters, 112, Article ID: 
153601. https://doi.org/10.1103/PhysRevLett.112.153601

[15] Harris, R.K., Becker, E.D., de Menezes, S.M.C., Goodfellow, R. and Granger, P. (2002) NMR Nomenclature: Nuclear Spin Properties and Conventions for Chemical Shifts (IUPAC Recommendations 2001). Annals of Magnetic Resonance, 1, 43-64. http://publications.iupac.org/pac/2001/7311/7311x1795.html

[16] McBride, N., Bland, P.A. and Gilmour, I. (2004) An Introduction to the Solar System. Cambridge University Press, Cambridge, 248.

[17] Franke-Arnold, S. Optical Angular Momentum and Atoms. https://royalsocietypublishing.org/doi/10.1098/rsta.2015.0435

[18] Gasiorowicz, S. and Langacker, P. (2005) Elementary Particles in Physics. https://www.physics.upenn.edu/ pgl/e27/E27.pdf

[19] Fernandez, E. (2006) Neutrino Physics at Accelerators. Journal of Physics. Conference Series, 53, 83-106. https://doi.org/10.1088/1742-6596/53/1/004

[20] Padgett, M. and Alen, L. (2000) Light with a Twist in Its Tail. Contemporary Physics, 41, 275-285.

https://www.tandfonline.com/doi/abs/10.1080/001075100750012777 https://doi.org/10.1080/001075100750012777

[21] Hessels, J.W.T., Ransom, S.M., Stairs, I.H., Freire, P.C., Kaspi, V.M. and Camilo, F. (2006) A Radio Pulsar Spinning at 716 Hz. Science, 311, 1901-1904.

https://doi.org/10.1126/science.1123430

[22] Lin, C.C. and Shu, F.H. (1964) On the Spiral Structure of Disk Galaxies. Astrophysical Journal, 140, 646. https://doi.org/10.1086/147955

[23] Francis, C. and Canderson, E. (2009) Galactic Spiral Structure. Proceedings of the Royal Society A: Mathematical, Physical and Engineering Sciences, 465, 3425-3446. https://doi.org/10.1098/rspa.2009.0036

[24] Dawson, R.I. and Johnson, J.A. (2018) Origins of Hot Jupiters. Annual Review of Astronomy and Astrophysics, 56, 175-221.

https://doi.org/10.1146/annurev-astro-081817-051853

[25] Wang, J., Fischer, D.A., Horch, E.P. and Huang, X. (2015) On the Occurrence Rate of Hot Jupiters in Different Stellar Environments. The Astrophysical Journal, 799, 229. https://doi.org/10.1088/0004-637X/799/2/229

[26] Schneider, J. Interactive Extra-Solar Planets Catalog. The Extrasolar Planets Encyclopedia. http://exoplanet.eu/catalog.php

[27] https://apod.nasa.gov/apod/ap121205.html

[28] A Remarkably Large Depleted Core in the Abell 2029 BCG IC 1101. https://researchbank.swinburne.edu.au/file/5bdd309d-145f-47d4-80e9-1a3591f7c51 e/1/2017-dullo-a_remarkably_large.pdf

[29] The Giant Elliptical Galaxy ESO 306-17. https://esa.int/ESA Multimedia/Images/2010/03/

[30] Hunter, D.A., Elmegreen, B.C., Rubin, V.C., Ashburn, A., Wright, T., Jozsa, G.I.G. and Struve, C. (2013) Star Formation in Two Luminous Spiral Galaxies. The Astronomical Journal, 146, 92.

https://iopscience.iop.org/article/10.1088/0004-6256/146/4/92 https://doi.org/10.1088/0004-6256/146/4/92

[31] Cortese, L., Marcillac, D., Richard, J., Bravo-Alfaro, H., Kneib, J-P., Rieke, G., Covone, G., Egami, E., Rigby, J., Czoske, O. and Davies, J. (2006) The Strong Transformation of Spiral Galaxies in-falling into Massive Clusters at $\mathrm{z} \sim 0.2$. Cambridge University Press, Cambridge. https://doi.org/10.1017/S1743921306006041 
[32] https://www.nasa.gov/mission_pages/galex/galex20130110.html

[33] Eufrasio, R.T., Dwek, E., Arendt, T.G., de Mello, D.F., Gadotti, D.A., Urrutia-Viscarra, F., de Oliveira, C.M. and Benform, DJ. (2014) Star Formation Histories Across the Interacting Galaxy NGC 6872, the Largest-Known Spiral. The Astrophysical Journal, 795, 89. https://doi.org/10.1088/0004-637X/795/1/89

[34] Loubser, S.I., Sansom, A.E., Sanchez-Blazquez, P., Soechting, I.K. and Bromage, G.E. (2008) Radial Kinematics of Brightest Cluster Galaxies. Monthly Notices of the Royal Astronomical Society, 391, 1009-1028. https://doi.org/10.1111/j.1365-2966.2008.13813.x

[35] Jog, C.J. (2002) Large Scale Asymmetry of Rotation Curves in Lopsided Spiral Galaxies. Astronomy and Astrophysics, 391, 471-479.

https://doi.org/10.1051/0004-6361:20020832

[36] Longo, M.J. (2011) Detection of a Dipole in the Handedness of Spiral Galaxies with Redshifts z 0.04. Physics Letters B, 699, 224-229.

[37] Sivaram, C. and Arun, K. Primordial Rotation of the Universe and Angular Momentum of a Wide Range of Celestial Objects. https://arxiv.org/pdf/1111.3873

[38] Morsch, O. and Arimondo, E. (2002) Ultracold Atoms and Bose-Einstein Condensate in Optical Lattices. In: Dauxois, T., Ruffo, S., Arimondo, E. and Wilkens, M., Eds., Dynamics and Thermodynamics of Systems with Long-Range Interactions, Lecture Notes in Physics, Vol. 602, Springer, Berlin, Heidelberg, 312-331.

https://doi.org/10.1007/3-540-45835-2_10

[39] Sachdev, S. (2011) Quantum Phase Transition from a Superfluid to a Mott Insulator in a Gas of Ultracold Atoms. Cambridge University Press, Cambridge. 